\title{
TDP-43: A RELIABLE IMMUNOHISTOCHEMISTRY MARKER FOR INCLUSION BODY MYOSITIS?
}

\author{
ASHOK VERMA, MD, DM* and RUP TANDAN, MD, FRCP\# \\ *Department of Neurology, University of Miami Miller School of Medicine, \\ Clinical Research Building, 1120 NW 14 Street, Suite 1317 Miami, FL 33136 \\ \#Department of Neurology, University of Vermont College of Medicine, \\ Room C225 Given Building, 89 Beaumont Avenue, Burlington, VT 05405
}

TDP-43 is a 414 amino acid protein encoded by the transactive response (TAR) DNA binding protein gene on chromosome $1 \mathrm{p} 36$. It was first cloned as a human protein capable of binding to the TAR DNA of HIV-1. ${ }^{11}$ It was later identified as part of a complex involved in splicing of the cystic fibrosis transmembrane conductance regulator gene. ${ }^{3}$ TDP-43 is highly conserved, ubiquitously expressed and predominantly localized to the nucleus under normal conditions. It consists of two RNA recognition motifs and a glycine-rich C-terminal region which interacts with other members of the heterogeneous ribonucleoprotein (hnRNP) family. ${ }^{2,5}$ TDP-43 is chiefly involved in well characterized transcription function and RNA splicing regulation. Recent studies suggest that TDP-43 may be involved in other cellular processes, such as microRNA biogenesis, apoptosis, cell division, mRNA stabilization and regulation of neuronal plasticity. ${ }^{2,5}$ Since its link to sporadic amyotrophic lateral sclerosis (sALS), frontotemporal dementia (FTD) with ubiquitinated protein inclusions (UBIs) (FTD-U) and FTD-U/ALS in 2006, overexpression of TDP-43 has been reported in other conditions with cellular overexpression, including Guaminian ALS-parkinsonism-dementia complex, progranulin gene mutation, VCP (Valosin-containing protein) gene mutation, dementia linked to chromosome 9 without VCP gene mutation, sporadic and hereditary IBM, and myofibrillar and desmin myopathies. ${ }^{6,7,9,12,14,15}$ Earlier this year, two independent researchers 8,16 reported that mutations in the gene for another DNA/RNA binding protein, fused in sarcoma or translocation in liposarcoma (FUS/TLS), also trigger UBIs and premature degeneration of motor neurons. FUS/TLS protein has not

Correspondence to: Rup Tandan, e-mail: rup.tandan@uvm.edu

(C) 2009 Wiley Periodicals, Inc.

Published online 15 June 2009 in Wiley InterScience (www.interscience.wiley. com). DOI 10.1002/mus. yet been studied in IBM. Nevertheless, alteration in RNA processing and protein aggregates appears to be the key event in these motor neuron and muscle diseases.

Sporadic inclusion body myositis (sIBM) is a common form of inflammatory muscle disease in older patients. In the first detailed pathological report of sIBM, ${ }^{4}$ Carpenter et al. commented on the characteristic inclusions as well as the nuclear degenerative process. Subsequent investigations (reviewed by Oldfors and Lindberg ${ }^{10}$ ) revealed similar inclusions - UBIs - in both dominant and recessive forms of hereditary IBM (hIBM), oculopharyngeal muscular dystrophy, and in IBM with Paget disease of bone and frontotemporal dementia.

Interestingly, when protein epitopes of UBIs in muscle fibers of sIBM were further investigated, it became clear that several "alien" molecules were overexpressed. These included $\beta$-amyloid, phosphorylated tau and SMI-31 that incidentally were also the constituents of UBIs in the neurons of Alzheimer disease brains. These observations prompted Banwell and Engel ${ }^{1}$ to search for other protein molecules that were overexpressed in astrocytes and astroglial cells in the vicinity of senile plaques of Alzheimer disease brains. One such molecule - $\alpha \mathrm{B}$ crystallin, indeed, was found to be overexpressed in sIBM. ${ }^{1}$ The demonstration of either immunoreactivity with antibodies to ubiquitin, SMI-31 or $\alpha \mathrm{B}$-crystallin, or the finding of Congo red fluorescence ( $\beta$-amyloid) then became the standard procedures for the histological diagnosis of IBM. Over the past year, TDP-43 immunoreactive UBIs have been reported in sIBM $^{7,15}$ and in familial myopathies with or without vacuolar change. ${ }^{7,12,15}$

In this issue of Muscle and Nerve, Salajegheh and colleagues ${ }^{13}$ confirm and extend the recent observations ${ }^{7,12,15}$ of excessive TDP-43 in IBM muscle fibers. These findings are important on several counts. First, the authors found an average of nearly 1 in 4 
IBM muscle fibers were immunoreactive to sarcoplasmic TDP-43. This makes the TDP-43 marker positivity a far more frequent finding than the presence of rimmed vacuoles, fluorescent Congo red material or other previously described markers (mostly in less than $1 \%$ of fibers). Second, TDP-43 inclusions were also demonstrated in muscle fibers of hIBM and myofibrillar myopathies. Coupled with similar findings in other recent reports, ${ }^{7,12,15}$ this suggests a common element in the pathogenesis of these myopathies. An occasional PM case $^{13}$ also showed TDP-43 positive muscle fibers; whether it represented atypical IBM remains uncertain. Taken together, as the authors surmise, TDP-43 is so far the most sensitive and specific diagnostic immunohistochemistry marker for IBM. Third, no TDP-43 gene mutation was discovered in this report, although only $6 \mathrm{sIBM}$ samples were investigated for direct sequencing of the coding sequence and exon-intron splicing sites of the TDP-43 gene. Although it would be premature to draw conclusions without additional data, it appears that a TDP-43 mutation is not the underlying cause in many sIBM patients. One should be extra mindful of this observation, as no TDP-43 gene mutation was initially discovered in a small cohort of sALS cases. ${ }^{6}$ However, it is now well known that TDP-43 mutations have been identified in fALS and sALS cases. ${ }^{14}$

The significance of these reproducible TDP-43 inclusions in IBM muscle is presently unknown. It is unclear whether the TDP-43 aggregates in IBM are just trashed proteins, or whether they are pathogenic through either gain of one or more toxic properties or loss of normal function from its sequestration in the cytoplasm and corresponding disruption of its interaction with nuclear protein partners and RNA targets. The abnormal redistribution of TDP- 43 from the nucleus to the cytoplasm in affected IBM muscle fibers is reminiscent of similar findings in FTD-U, sALS, and the recently described TDP-43 yeast model. ${ }^{5}$ It is also unclear if factors that affect the normal intracellular trafficking of TDP-43, from the cytoplasm to the nucleus, predispose to both the formation of abnormal aggregates (inclusions) and the loss of nuclear localization.

The findings in the current report are potentially very important. The striking specificity and high degree of sensitivity, in concert with other recent reports, $7,12,15$ could make TDP-43 a standard diagnostic marker for IBM. A number of questions remain unanswered, however. It is unknown if protein aggregates are a cause or consequence of the disease process. It will be interesting to see if FUS/TLC aggregates also occur in IBM muscle fibers, and if mutations in any of the DNA/RNA binding proteins (TDP-43, FUS/TLC, and others) are discovered in familial or sporadic IBM. Both cellular and animal models will be essential to define the link between TDP-43 and IBM. Finally, a better understanding of the role of TDP-43 in IBM may be crucial to the development of targeted therapeutic strategies in IBM.

\section{REFERENCES}

1. Banwell BL, Engel AG. $\alpha$ B-crystalline immunolocalization yields new insights into inclusion body myositis. Neurology. 2000;54:1033-1041.

2. Buratti E, Baralle FE. Multiple roles of TDP-43 in gene expression, splicing regulation, and human disease. Front Biosci. 2008;13:867-878.

3. Buratti E, Dörk T, Zuccato E, Pagani F, Romano M, Baralle FE. Nuclear factor TDP-43 and SR proteins promote in vitro and in vivo CFTR exon 9 skipping. EMBO J. 2001;20(7):1774-1784.

4. Carpenter S, Karpati G, Heller I, Eisen A. Inclusion body myositis: a distinct variety of idiopathic inflammatory myopathy. Neurology. 1978;28:8-17.

5. Johnson BS, McCaffery JM, Lindquist S, Gitler AD. A yeast TDP-43 proteinopathy model: Exploring the molecular determinants of TDP-43 aggregation and cellular toxicity. Proc Natl Acad Sci U S A. 2008;105:6439-6444.

6. Gijselinck I, Sleegers K, Engelborghs S, Robberecht W, Martin JJ, Vandenberghe R, et al. Neuronal inclusion protein TDP-43 has no primary genetic role in FTD and ALS. Neurobiol Aging. 2007 Dec 7 [Epub ahead of print].

7. Küsters B, van Hoeve BJ, Schelhaas HJ, Ter Laak H, van Engelen BG, Lammens M. TDP-43 accumulation is common in myopathies with rimmed vacuoles. Acta Neuropathol. 2009;117:209-211.

8. Kwiatkowski TJ Jr, Bosco DA, Leclerc AL, Tamrazian E, Vanderburg CR, Russ C, et al. Mutations in the FUS/TLS gene on chromosome 16 cause familial amyotrophic lateral sclerosis. Science. 2009;323:1205-1208.

9. Neumann M, Sampathu DM, Kwong LK, Truax AC, Micsenyi MC, Chou TT, et al. Ubiquitinated TDP-43 in frontotemporal lobar degeneration and amyotrophic lateral sclerosis. Science. 2006;314:130-133.

10. Oldfors A, Lindberg C. Diagnosis, pathogenesis and treatment of inclusion body myositis. Curr Opin Neurol. 2005;18: 497-503.

11. Ou SH, Wu F, Harrich D, García-Martínez LF, Gaynor RB. Cloning and characterization of a novel cellular protein, TDP43, that binds to human immunodeficiency virus type 1 TAR DNA sequence motifs. J Virol. 1995;69:3584-3596.

12. Olivé M, Janué A, Moreno D, Gámez J, Torrejón-Escribano B, Ferrer I. TAR DNA-Binding protein 43 accumulation in protein aggregate myopathies. J Neuropathol Exp Neurol. 2009; 68:262-273.

13. Salajegheh M, Pinkus JL, Taylor JP, Amato AA, Nazareno R, Baloh RH, Greenberg SA. Sarcoplasmic redistribution of nuclear TDP-43 in inclusion body myositis. Muscle Nerve. 2009; 40(1):19-31.

14. Sreedharan J, Blair IP, Tripathi VB, Hu X, Vance C, Rogelj B, et al. TDP-43 mutations in familial and sporadic amyotrophic lateral sclerosis. Science. 2008;319:1668-1672.

15. Weihl CC, Temiz P, Miller SE, Watts G, Smith C, Forman M, et al. TDP-43 accumulation in inclusion body myopathy muscle suggests a common pathogenic mechanism with frontotemporal dementia. J Neurol Neurosurg Psychiatry. 2008;79: 1186-1189.

16. Vance C, Rogelj B, Hortobágyi T, De Vos KJ, Nishimura AL, Sreedharan J, et al. Mutations in FUS, an RNA processing protein, cause familial amyotrophic lateral sclerosis type 6 . Science. 2009;323:1208-1211. 